University of Nebraska - Lincoln

DigitalCommons@University of Nebraska - Lincoln

$11-2008$

\title{
Flush-Mounted Steady-Periodic Heated Film With Application to Shear-Stress Measurement
}

Kevin D. Cole

University of Nebraska-Lincoln, kcole1@unl.edu

Follow this and additional works at: https://digitalcommons.unl.edu/mechengfacpub

Part of the Mechanical Engineering Commons

Cole, Kevin D., "Flush-Mounted Steady-Periodic Heated Film With Application to Shear-Stress Measurement" (2008). Mechanical \& Materials Engineering Faculty Publications. 48.

https://digitalcommons.unl.edu/mechengfacpub/48

This Article is brought to you for free and open access by the Mechanical \& Materials Engineering, Department of at DigitalCommons@University of Nebraska - Lincoln. It has been accepted for inclusion in Mechanical \& Materials Engineering Faculty Publications by an authorized administrator of DigitalCommons@University of Nebraska Lincoln. 


\section{Flush-Mounted Steady-Periodic Heated Film With Application to Shear-Stress Measurement}

\author{
Kevin D. Cole \\ Mechanical Engineering Department, \\ University of Nebraska-Lincoln, \\ Lincoln, NE 68588-0656 \\ e-mail: kcole1@unl.edu
}

Surface-mounted heated films have been used for fluid-flow measurement for many years. Recently unsteady heating of such surface films has been explored experimentally. In this paper steady-periodic heating of a surface-mounted film is studied analytically. Wall effects and axial heat conduction in the fluid are included. The temperature is found as an exact integral expression constructed from separate Green's function formulations in the fluid flow and in the solid wall that are matched at the fluid-solid interface. Results for temperature, obtained by quadrature, are reported for several flow speeds and several steady-periodic frequencies. The results show that steady-periodic heating has potential for shear-stress measurement because the heating frequency may be tuned to maximize the temperature response at the shear stress of interest. Thermal calibration of these sensors is discussed, and simulated calibration curves are given.

[DOI: 10.1115/1.2955472]

Keywords: hot-film anemometer, gas flow, frequency response, conjugate heat transfer, thermal wave

\section{Introduction}

Flush-mounted thermal sensors have been used for shear-stress measurements for many years with steady heating [1]. Recently pulsed heating has been investigated experimentally for obtaining quantitative shear-stress values [2] and observing shear-stress direction [3].

The purpose of this paper is to explore steady-periodic heating of flush-mounted hot-film sensors with application to shear-stress measurement. The pertinent literature will be discussed next in the areas of flush-mounted heated films, conjugate heat transfer, and periodic heating applied for thermal measurements.

There have been several theoretical studies of heat/mass transfer from flush-mounted sensors to a fluid with no participating wall, which exactly describes mass-transfer sensors and is a good approximation for thermal sensors in liquids. The steady response of a circular mass-transfer sensor was studied with a boundaryelement solution by Stone [4] and with an asymptotic method by Phillips [5]. Soliman and Chambre [6] used a double Laplace transform to find the response of a steady fluid flow to a step change in boundary heating; Geshev [7] reported theoretical results for the response of a strip-shaped sensor to a fluid flow containing a small sinusoidal oscillation; Steenhoven and van de Beucken [8] reported numerical results for a strip-shaped sensor and experimental results were reported for a mass-transfer sensor, which was circular in shape. The flow contained a low-frequency oscillating component over a range of amplitudes, the largest of which caused flow reversals.

The phrase "conjugate heat transfer" is used to describe convection heat transfer with a participating wall. The temperature and heat flux at the fluid-solid boundary are initially unknown and must be determined from the solution of the entire problem. The author has previously investigated steady conjugate heat transfer from a flush-mounted film [9]. Although the work was limited to high Peclet number (axial conduction in the fluid was neglected),

Contributed by the Heat Transfer Division of ASME for publication in the JourNal of Heat Transfer. Manuscript received September 10, 2007; final manuscript received January 16, 2008; published online August 29, 2008. Review conducted by Minking Chyu. Paper presented at the 2006 ASME International Mechanical Engineering Congress (IMECE2006), Chicago, IL, November 5-10, 2006. a wide variety of flow conditions, fluid-solid property ratios, and wall thicknesses were studied. The solution involved distinct Green's function (GF) descriptions of the temperature in the fluid and the solid, matched numerically at the interface. In a later study, axial heat conduction in the fluid was included and an exact integral expression was found for the steady-temperature solution [10]. Quadrature was used to obtain numerical results. An asymptotic method was used by Stein et al. [11] to study the steady-temperature decay downstream of a strip heater on a slab wall. Chao et al. [12] used a finite-difference method to compute temperature and velocity contours near a very small heater $(a$ $=0.2 \mathrm{~mm}$ ) on a silicon substrate cooled by airflow. Axial conduction of the fluid is important when the heater is very small.

There have been several studies of unsteady conjugate heat transfer. Cole and Beck [13] simulated a strip-shaped film and a single on-off pulse of heat. Later Liang and Cole [14] extended this work to a 3D rectangular flush-mounted sensor. In these studies a simplified fluid-side theory was used to represent flow with large Peclet number (axial heat conduction neglected in the fluid) and low-frequency thermal transients (as the fluid heat transfer was quasisteady). A later study by the author included axial heat conduction in the fluid and a rapid heating event [15]. Dinu et al. [16] simulated the wall-side heat conduction from a flushmounted heater for use as a heat flux sensor. The fluid flow was modeled as a convection boundary condition on the wall. Frequency-response results show that the phase depends only on the excitation frequency and not on the signal size. Several papers report studies of a heater with specified temperature (such as provided by a constant-temperature anemometer circuit) with a transient caused by variations in the flow velocity [17-20]. The intent of these papers was to find the frequency response of the sensor to fluid-flow transients. Of particular note in this group is Tardu and Pham [20] whose two-dimensional numerical study showed that axial fluid conduction must be included when the sensor is small and when the fluid flow is small. They also showed that measurements in gas flows are the most challenging because of heat transfer to the wall.

There have been many studies of steady-periodic heating applied to measurement of thermal properties in solids, including the thermal hot-strip method [21,22], the 3-omega method [23], and photothermal methods $[24,25]$. 


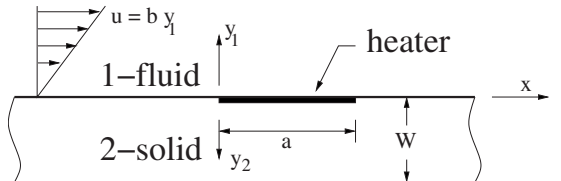

Fig. 1 Geometry for a flush-mounted heated film cooled by a fluid flow

Recently there have been a few studies of steady-periodic heating effects applied to fluid-flow measurement. Bonne and Kubisiak [26] have developed a mechanical actuator combined with two identical flush-mounted thermal sensors. The mechanical actuator produces steady-periodic reversing flow over one thermal sensor to calibrate the main thermal sensor for measurement of flow velocity for any flowing liquid. Al-Salaymeh and Durst [27] studied gas flow and Chung et al. [28] studied liquid flow using a single-wire sensor energized by a periodic heating signal. The same wire is also used as a temperature sensor, and the phase lag of the temperature response is combined with a transient model of the heated wire to deduce the fluid-flow velocity around the wire. This type of sensor has a wider velocity range than a wire energized by a traditional constant-temperature anemometer circuit. Rachalski [29] used a three-wire system with one periodically heated wire and two temperature-sensing wires. The temperature signal was analyzed using a GF solution for the heat transfer in a uniform-velocity flow.

To the author's knowledge steady-periodic heating has not been applied for the measurement of wall shear stress. This paper is a theoretical study of a periodically heated film, flush mounted on a solid wall, applied to the measurement of shear stress. This paper is divided into sections on the temperature equations, the GF, theory for two regions in contact, numerical results, and a summary.

\section{Temperature Equations}

The equations describing the temperature in the fluid and the solid are given in this section. The temperature distribution $\widetilde{T}(x, y, t)$ satisfies

$$
\begin{gathered}
\frac{\partial^{2} \widetilde{T}_{1}}{\partial x^{2}}+\frac{\partial^{2} \widetilde{T}_{1}}{\partial y_{1}^{2}}=\frac{b y_{1}}{\alpha_{1}} \frac{\partial \widetilde{T}_{1}}{\partial x}+\frac{1}{\alpha_{1}} \frac{\partial \widetilde{T}_{1}}{\partial t}, \quad 0<y_{1}<\infty \\
\frac{\partial^{2} \widetilde{T}_{2}}{\partial x^{2}}+\frac{\partial^{2} \widetilde{T}_{2}}{\partial y_{2}^{2}}=-\frac{1}{k_{2}} \widetilde{g}\left(x, y_{2}, t\right)+\frac{1}{\alpha_{2}} \frac{\partial \widetilde{T}_{2}}{\partial t} \quad \text { in domain } \Omega_{2}
\end{gathered}
$$

The fluid velocity is described by a linear distribution $b y_{1}$ because the thermal boundary layer is contained in the near-wall portion of the fluid velocity boundary layer [9]. Quantity $\tilde{g}$ is the volume energy source in Region 2 that describes any heating condition including a flush-mounted heated film. Domain $\Omega_{2}$ can include the slab body $\left(0<y_{2}<W\right)$ as shown in Fig. 1 or the semi-infinite body $\left(0<y_{2}<\infty\right)$.

The boundary conditions for the combined domain are the following:

$$
\begin{gathered}
\text { at } x \rightarrow \pm \infty, \quad \tilde{T}_{i} \text { is bounded, } \quad i=1,2 \\
\text { at } y_{1} \rightarrow \infty, \quad \widetilde{T}_{1} \text { is bounded } \\
\text { at } y_{2}=W, \quad k \frac{\partial \widetilde{T}_{2}}{\partial y_{2}}+h \widetilde{T}_{2}=\tilde{f}_{2}(x, t)
\end{gathered}
$$

The boundary condition at $y_{2}=W$ may be one of the three types depending on the values of coefficients $k$ and $h$ : boundary type 1 is specified temperature for $k=0$ and $h=1$, boundary type 2 is specified heat flux for $h=0$, and boundary type 3 is specified convection.

Since in this paper the applications are limited to steadyperiodic heating, we take the heating terms $\widetilde{g}$ and $\tilde{f}$ and the resulting temperature $\widetilde{T}_{i}(x, y, t)$ to be steady periodic at a single frequency. That is, let

$$
\begin{gathered}
\tilde{g}(x, y, t)=\operatorname{Re}\left[g(x, y, \omega) e^{j \omega t}\right] \\
\tilde{f}(x, t)=\operatorname{Re}\left[f(x, \omega) e^{j \omega t}\right] \\
\widetilde{T}_{i}(x, y, t)=\operatorname{Re}\left[T_{i}(x, y, \omega) e^{j \omega t}\right]
\end{gathered}
$$

Now in Eqs. (1)-(3) we replace $\widetilde{g}, \widetilde{f}$, and $\widetilde{T}_{i}$ with $g e^{j \omega t}, f e^{j \omega t}$, and $T_{i} e^{j \omega t}$, respectively, to find the steady-periodic equations

$$
\begin{gathered}
\frac{\partial^{2} T_{1}}{\partial x^{2}}+\frac{\partial^{2} T_{1}}{\partial y_{1}^{2}}=\frac{b y_{1}}{\alpha_{1}} \frac{\partial T_{1}}{\partial x}+\frac{j \omega}{\alpha_{1}} T_{1} \\
\frac{\partial^{2} T_{2}}{\partial x^{2}}+\frac{\partial^{2} T_{2}}{\partial y_{2}^{2}}=-\frac{1}{k_{2}} g\left(x, y_{2}, \omega\right)+\frac{j \omega}{\alpha_{2}} T_{2} \\
\left.k \frac{\partial T_{2}}{\partial y_{2}}\right|_{y_{2}=W}+\left.h T_{2}\right|_{y_{2}=W}=f(x, \omega)
\end{gathered}
$$

In this paper complex-valued $T_{i}(x, y, \omega)$ is interpreted as the steady-periodic temperature (Kelvin) in the $i$ th body ( $i=1$ or 2$)$ at a single frequency $\omega$. For further discussion of this point, see Ref. [30], pp. 2 and 3. Later in the paper, results will be discussed in the form of amplitude and phase of this temperature.

\section{GF Solution in Each Region}

The steady-periodic temperature will be stated formally with the GF method. Assume for the moment that the GF in frequency space, $G$, is known. Then the steady-periodic temperature is given by the following integral equation (see Ref. [31], pp. 40-43):

$$
T_{i}(x, y, \omega)=\frac{\alpha_{i}}{k_{i}} \int g\left(x^{\prime}, y_{i}^{\prime}, \omega\right) G_{i}\left(x-x^{\prime}, y_{i}, y_{i}^{\prime}, \omega\right) d x^{\prime} d y_{i}^{\prime}
$$

(for volume heating)

$$
+\alpha_{i} \int_{S} f\left[\begin{array}{cc}
-\partial G_{i} / \partial n^{\prime} & \text { (type } 1 \text { only) } \\
\frac{1}{k_{i}} G_{i} & \text { (type } 2 \text { or } 3)
\end{array}\right] d s^{\prime}
$$

(at the boundaries)

for regions $i=1,2$. Here $S$ is the surface area and $n$ is the outward normal unit vector on the boundary. The same GF appears in each integral term but it is evaluated at locations appropriate for each integral. The GF's needed in the above equation are discussed in detail in the appendixes. Next the temperature solution for two regions in contact will be examined.

\section{Two Regions in Contact}

In this section the thermal response of two regions in contact will be studied to model conjugate heat transfer with application to shear-stress measurement. Consider two regions in contact as shown in Fig. 1.

Let Region 2 be a stationary solid heated by a known volume energy source, $g(x, y, \omega)$, and let Region 1 be a flowing fluid, which is heated by contact with Region 2 . Then the temperatures in each region may be formally stated with the GF solution equation given in Eq. (8), in terms of known functions $G_{1}$ and $G_{2}$ and unknown interface heat fluxes $q_{1}$ and $q_{2}$, as follows:

$$
T_{1}\left(x, y_{1}, \omega\right)=\frac{\alpha_{1}}{k_{1}} \int q_{1}\left(x^{\prime}, \omega\right) G_{1}\left(x-x^{\prime} y_{1}, \omega\right) d x^{\prime}
$$




$$
T_{2}\left(x, y_{2}, \omega\right)=\frac{\alpha_{2}}{k_{2}} \int q_{2}\left(x^{\prime}, \omega\right) G_{2}\left(x-x^{\prime}, y_{2}, \omega\right) d x^{\prime}+B_{2}\left(y_{2}\right)
$$

where

$$
B_{2}\left(y_{2}\right)=\frac{\alpha_{2}}{k_{2}} \iint g\left(x^{\prime}, y_{2}^{\prime}, \omega\right) G_{2}\left(x-x^{\prime}, y_{2}, y_{2}^{\prime}\right) d x^{\prime} d y_{2}^{\prime}
$$

Here $B_{2}$ is the contribution to the temperature caused by the volume energy source. The volume energy source, $g$, could represent any spatial distribution, such as from a thin heater at the surface.

Next consider matching conditions at the interface between the regions. The heat flux entering Region 1 leaves Region 2, and the temperatures match at the interface. That is,

$$
\begin{gathered}
q_{1}(x, \omega)=-q_{2}(x, \omega) \\
T_{1}(x, 0, \omega)=T_{2}(x, 0, \omega)
\end{gathered}
$$

The temperature solution will be sought with a spatial-Fourier transform defined by the following transform pair:

$$
\begin{gathered}
\bar{T}(\beta)=\int_{-\infty}^{\infty} T(x) e^{-j \beta x} d x \\
T(x)=\frac{1}{2 \pi} \int_{-\infty}^{\infty} \bar{T}(\beta) e^{j \beta x} d \beta
\end{gathered}
$$

Apply the integral transform to Eqs. (9)-(12) to obtain

$$
\begin{gathered}
\bar{T}_{1}\left(\beta, y_{1}, \omega\right)=\frac{\alpha_{1}}{k_{1}} \bar{q}_{1}(\beta, \omega) \bar{G}_{1}\left(\beta, y_{1}, y_{1}^{\prime}=0, \omega\right) \\
\bar{T}_{2}\left(\beta, y_{2}, \omega\right)=\frac{\alpha_{2}}{k_{2}} \bar{q}_{2}(\beta, \omega) \bar{G}_{2}\left(\beta, y_{2}, y_{2}^{\prime}=0, \omega\right)+\bar{B}_{2}\left(y_{2}\right) \\
\bar{q}_{1}(\beta, \omega)=-\bar{q}_{2}(\beta, \omega) \\
\bar{T}_{1}(\beta, 0, \omega)=\bar{T}_{2}(\beta, 0, \omega)
\end{gathered}
$$

Note that the convolution rule has been used to strip away the integral over $x^{\prime}$. Now evaluate temperatures $\bar{T}_{1}$ and $\bar{T}_{2}$ at the interface and substitute them into Eq. (18). Supressing the dependence on $\beta$ and $\omega$, Eq. (18) becomes

$$
\frac{\alpha_{1}}{k_{1}} \bar{q}_{1} \bar{G}_{1}=\frac{\alpha_{2}}{k_{2}} \bar{q}_{2} \bar{G}_{2}+\bar{B}_{2}(0)
$$

Now define $\phi_{i}=\left(\alpha_{i} / k_{i}\right) \bar{G}_{i}\left(y_{i}=y_{i}^{\prime}=0\right)$, eliminate $\bar{q}_{2}$ with Eq. (17), and solve the above equation for $\bar{q}_{1}$ as follows:

$$
\bar{q}_{1}=\frac{\bar{B}_{2}(0)}{\phi_{1}+\phi_{2}}
$$

The above expression for $\bar{q}_{1}$ represents an exact solution to the problem in $\beta$-space, because it can be replaced with Eqs. (15) or (16) to give the temperature solution anywhere in either region in $\beta$-space. Of course, the appropriate inverse transform is needed to evaluate the temperature in $x$-space. The above example has treated two regions in contact, however, this approach can be extended to any number of layers in contact for an exact solution in $\beta$-space (see, for example, Ref. [24]).

Response of Flush-Mounted Heater. In the special case of a thin, flush-mounted heater, the volume generation term takes the form $g\left(x, y_{2}, \omega\right)=q_{h}(x, \omega) \delta\left(y_{2}\right)$ where $q_{h}$ is the heater flux and $\delta$ is the Dirac delta function. Now replace this form of $g$ with the expression for $B_{2}$, evaluate the integral over $y_{2}$ with the sifting property of the Dirac delta function, and strip off the integral over $x^{\prime}$ with the spatial-Fourier transform

$$
\bar{B}_{2}\left(\beta, y_{2}, \omega\right)=\frac{\alpha_{2}}{k_{2}} \bar{q}_{h}(\beta, \omega) \bar{G}_{2}\left(\beta, y_{2}, \omega\right)
$$

Then, evaluate $\bar{B}_{2}$ at $y_{2}=0$ and replace the expression for the heat flux with the fluid, Eq. (20),

$$
\bar{q}_{1}=\frac{\bar{q}_{h} \phi_{2}}{\phi_{1}+\phi_{2}}
$$

Finally, replace this value for $\bar{q}_{1}$ with the expression for the fluid temperature as follows:

$$
\bar{T}_{1}\left(\beta, y_{1}, \omega\right)=\frac{\alpha_{1}}{k_{1}}\left(\frac{\bar{q}_{h} \phi_{2}}{\phi_{1}+\phi_{2}}\right) \bar{G}_{1}\left(\beta, y_{1}, \omega\right)
$$

This is the $\beta$-space temperature in the fluid caused by a flushmounted heater. The real-space temperature in the fluid is found with the inversion integral, Eq. (14),

$$
T_{1}\left(x, y_{1}, \omega\right)=\frac{1}{2 \pi} \int_{-\infty}^{\infty} \bar{T}_{1}\left(\beta, y_{1}, \omega\right) e^{j \beta x} d \beta
$$

Spatial Average on the Heater. The average temperature on the heater is a practical means to extract information from a heated film because the average temperature can be measured with suitable electronics such as that developed for pulse-heated thermal conductivity measurements [21]. The spatial-average temperature on the heater may be computed from the surface temperature by

$$
\begin{aligned}
T_{\mathrm{av}}(\omega) & =\frac{1}{a} \int_{0}^{a} T_{1}(x, 0, \omega) d x=\frac{1}{a} \int_{0}^{a}\left[\frac{1}{2 \pi} \int_{-\infty}^{\infty} \bar{T}_{1}(\beta, 0, \omega) e^{j \beta x} d \beta\right] d x \\
& =\frac{1}{2 \pi} \int_{-\infty}^{\infty} \bar{T}_{1}(\beta, 0, \omega)\left[\frac{\left(e^{j \beta a}-1\right)}{j \beta a}\right] d \beta
\end{aligned}
$$

Note that the spatial integral on $x$ has been carried out in closed form. Quantity $T_{\mathrm{av}}$ is actually easier to evaluate numerically, compared to $T_{1}(x)$, because the added factor of $1 / \beta$ causes the integrand to vanish more rapidly as $\beta \rightarrow \pm \infty$.

\section{Numerical Results}

In this section numerical results are given for heating caused by a flush-mounted, spatially uniform heater. Spatially uniform heating describes a thin metallic sensor whose temperature rise is small enough so that variation in the sensor electrical resistivity is likewise small. Another possible boundary condition, that of a spatially uniform temperature on the sensor, is not treated here. Let the spatially uniform heat-flux distribution be described by

$$
q_{h}(x, \omega)= \begin{cases}q_{0}, & 0<x<a \\ 0 & \text { otherwise }\end{cases}
$$

where $q_{0}$ is a constant. The spatial-Fourier transform of this heat flux is needed, found by applying Eq. (13) as follows:

$$
\bar{q}_{h}(\beta)=\int_{0}^{a} q_{h}(x, \omega) e^{-j \beta x} d x=q_{0} \frac{1-e^{-j \beta a}}{j \beta}
$$

This heating function is used for the numerical results discussed below.

Fluid Flow Alone. In this section calculations are reported for a fluid flow adjacent to a wall, which does not participate in the heat transfer. This is a useful limiting case, which can be checked against fluid-flow theory, and it also has application, by analogy, to certain mass-transfer sensors.

The temperature for the fluid-only case can be found from the two-region solution, Eq. (23), by taking the limit as $k_{2} \rightarrow 0$, which 


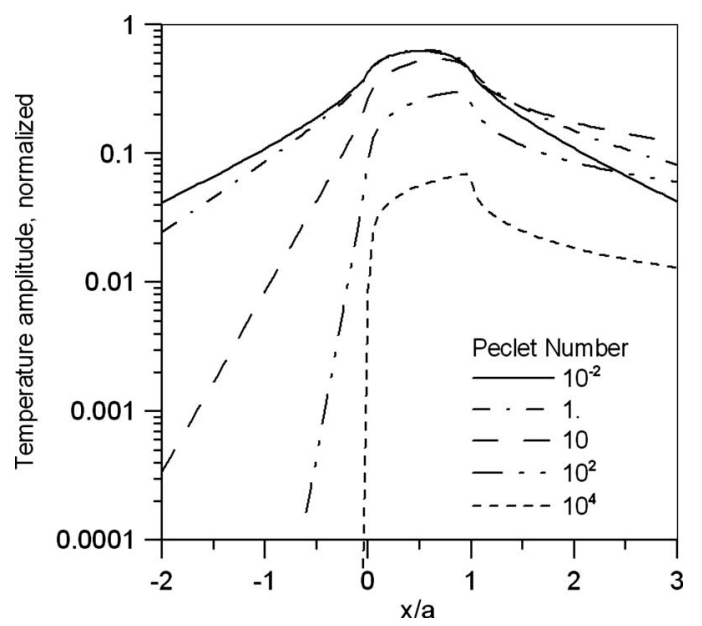

Fig. 2 Amplitude of temperature along the boundary of a flowing fluid heated over $0<x / a<1$ and insulated elsewhere for various flow values, at frequency $\omega^{+}=1$. No wall effects are present.

is consistent with no heat entering or leaving the wall. This causes $\phi_{2}$ to be much larger than $\phi_{1}$. Taking this limit in the temperature expression gives

$$
\begin{aligned}
\lim _{\phi_{2} \rightarrow \infty}\left[\bar{T}_{1}\left(\beta, y_{1}, \omega\right)\right] & =\frac{\alpha_{1}}{k_{1}}\left(\frac{\bar{q}_{h} \phi_{2}}{0+\phi_{2}}\right) \bar{G}_{1}\left(\beta, y_{1}, \omega\right) \\
& =\frac{\alpha_{1}}{k_{1}} \bar{q}_{h} \bar{G}_{1}\left(\beta, y_{1}, \omega\right)
\end{aligned}
$$

That is, the temperature reduces to that given by heating of the fluid alone.

Using the above expression for spatially uniform $\bar{q}_{h}$ and the expression for $\bar{G}_{1}$ from Appendix A, the $\beta$-space fluid temperature is given by

$$
\bar{T}_{1}\left(\beta, y_{1}, \omega\right)=\frac{\alpha_{1}}{k_{1}} \frac{q_{0}\left(e^{-j \beta a}-1\right)}{j \beta \alpha_{1} p^{1 / 3}} \frac{\operatorname{Ai}\left[\left(j \omega / \alpha_{1}+\beta^{2}+p y\right) / p^{2 / 3}\right]}{\operatorname{Ai}^{\prime}\left[\left(j \omega / \alpha_{1}+\beta^{2}\right) / p^{2 / 3}\right]}
$$

where $\mathrm{Ai}$ is the Airy function and where $p=b \beta j / \alpha_{1}$. The realspace temperature is obtained with the inverse-Fourier transform given by Eq. (24), which must be evaluated numerically.

Normalized parameters are used for presenting the results, as follows: The temperature is normalized as $T^{+}=\left(T k_{1}\right) /\left(q_{0} a\right)$ the frequency is normalized as $\omega^{+}=\omega a^{2} / \alpha_{1}$ and the normalized shear stress is given by the Peclet number $\left(\mathrm{Pe}=b a^{2} / \alpha_{1}\right)$. The Peclet number is defined with velocity gradient $b$, which is proportional to shear stress $\mu b$ where $\mu$ is viscosity. Using these parameters, Fig. 2 shows the amplitude of the temperature along the wall for a range of shear-stress values (Peclet number) and at frequency $\omega^{+}=1$. The fluid flow is from left to right. At $\mathrm{Pe}=10^{-2}$, the temperature amplitude is symmetric about the heated region, indicating that the $x$-direction heat conduction dominates the heat transfer and convection is negligible. One of the contributions of the present work is the retention of the axial conduction in the fluid, which allows for study of small-Pe conditions. The amplitude plot for the $\mathrm{Pe}=10^{-2}$ case shown in Fig. 2 has the same shape as that in earlier work by the author for heating of stationary solids [32].

As the Peclet number increases, the temperature on the heater decreases slightly, the upstream temperature falls precipitously, and the downstream temperature rises, all because of increasing convection heat transfer. At large Peclet number the fluid temperature is dominated by convection and $x$-direction conduction is negligible. Further, because frequency is small in Fig. 2, the re-

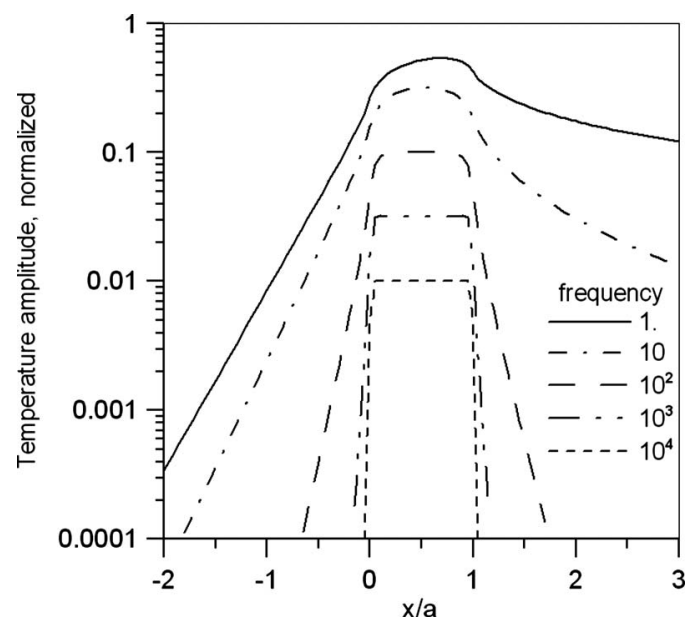

Fig. 3 Amplitude of temperature along the boundary of a flowing fluid heated over $0<x / a<1$ and insulated elsewhere for various frequencies, at fluid flow $\mathrm{Pe}=10$. No wall effects are present.

sults shown for $\mathrm{Pe}=10^{4}$ agree to four digits with steady convection-only theory [9], providing another check on the present results. This occurs because for Pe large and for small frequency, the time scale for convection is much shorter than the time scale for heating fluctuations.

Figure 3 shows the temperature amplitude at $\mathrm{Pe}=10$ for several heating frequencies. At $\omega^{+}=1$ the fluid temperature is quasisteady. The upstream region of the wall is dominated by conduction heat transfer and the warm region downstream represents a strong convection component. At $\omega^{+}=10$ the temperature amplitude drops slightly on the heater, but the temperature drops rapidly downstream where the convection component is much smaller. One explanation is that the higher frequency decreases the thermal penetration into the fluid so the steady-periodic thermal boundary layer is much thinner.

As the frequency increases to $\omega^{+}=10^{2}$, the downstream convection dissappears; however, $x$-direction conduction is still evident from the rounded temperature distribution at the edges of the heater. At $\omega^{+}=10^{3}$ and $10^{4}$ the temperature on the heater is flat, indicating that only $y$-direction conduction remains. That is, at high frequency, there remains only one-dimensional heat conduction perpendicular to the plane of the heater.

Next the spatial-average temperature on the heater will be discussed. Figure 4 shows the amplitude of average temperature on the heater versus Peclet number for several heating frequencies. At every frequency there is a range of low Peclet numbers for which the amplitude is flat. As Peclet increases, there is a point where the amplitude begins to vary with Peclet number. Note that the edge of the flat region occurs at higher Peclet numbers for higher frequency curves. This suggests that the shear-stress sensitivity for a particular sensor geometry can be tuned, by heating frequency, to the shear-stress level (Peclet level) of interest. An upper limit exists, however, as at higher frequencies the signal amplitude becomes smaller and smaller. As frequency increases in Fig. 4, a slight upward bump appears in the amplitude curve at the transition between flat and falling amplitudes. An echo of this feature also appears in the phase plot (Fig. 5) in the $\omega^{+}=100$ curve. At the present time the reason for this frequency-dependent feature is not understood.

Figure 5 shows the phase of the average heater temperature for the same conditions as for Fig. 4. The phase is important for several reasons. First, like the amplitude, the phase varies with the Peclet number in a clearly defined range determined by frequency. Second, and most important, the phase is an absolute quantity for which no calibration is needed. Phase measurements are not af- 


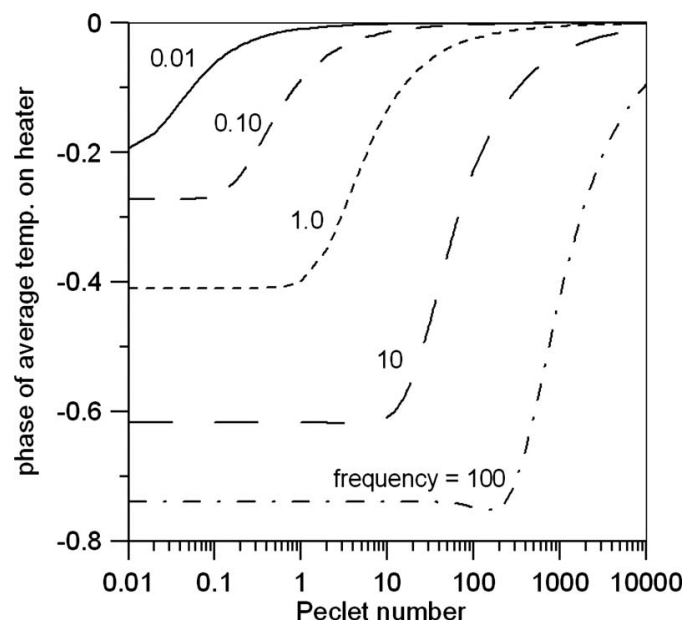

Fig. 5 Phase of spatial-average temperature on the heater for fluid flow alone, for various frequencies

fected by the "zero drift," which occurs in electronic amplifiers and which often appears in constant-temperature hot-film techniques. Finally, the sensitivity to Peclet number (slope of the curves in Fig. 5) actually increases with increasing frequency. In contrast, the amplitude sensitivity (slope in Fig. 4) is the same for every frequency. Clearly, the phase response is important for the flow-sensor application, especially at higher Peclet number.

The results given in this section have been for heat transfer in the fluid alone. In the next section the effects of heat conduction in the wall are included.

Gas Flow With Participating Wall. For thermal measurement of shear stress, gas flows are the most challenging because most of the introduced heat enters the wall, and only a small portion of the heat directly enters the fluid flow. For gas flows, then, the sensor signal contains a large wall-sensitive portion and a very small fluid-sensitive portion. Every steadily heated sensor design is limited by this wall-dominated response. Steady-periodic heating, however, combined with the use of the phase response of the sensor, can completely remove the steady signal from the measured response. The steady signal, which is removed, is primarily the wall response, greatly improving the sensitivity of the measurement to the fluid shear stress.

In this section, the two-body theory presented earlier is applied to find the steady-periodic temperature for a strip heater on a

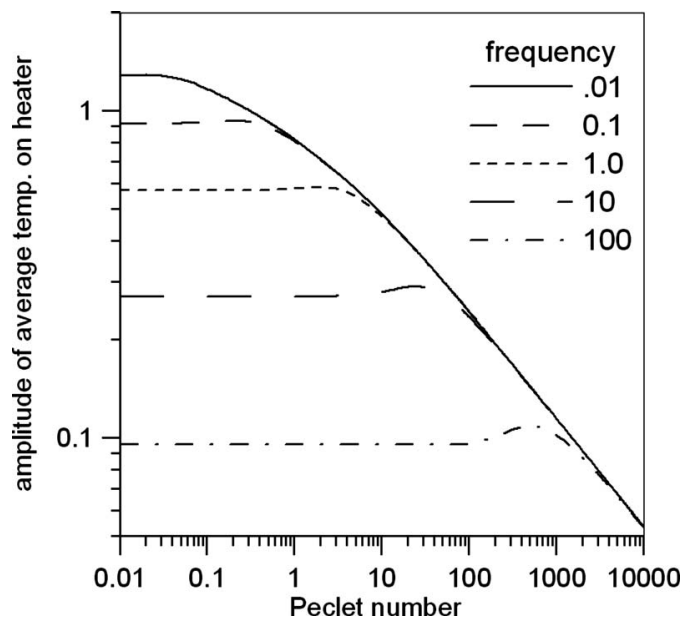

Fig. 4 Amplitude of spatial-average temperature on the heater for fluid flow alone, for various frequencies

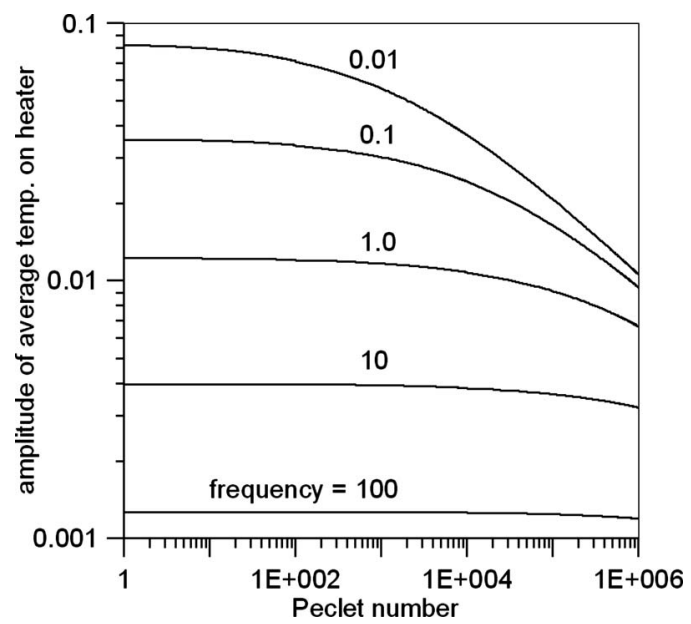

Fig. 6 Amplitude of spatial-average temperature on the heater for airflow over a polymer wall $\left(k_{1} / k_{2}=0.22, \alpha_{1} / \alpha_{2}=291\right)$ for various frequencies

thermally participating wall. Specifically, the interface temperature given by Eq. (25) is evaluated. Figure 6 shows the amplitude of the spatial-average temperature on the heater for $\alpha_{1} / \alpha_{2}=291$ and $k_{1} / k_{2}=0.22$ to simulate air flow over a kapton (polymer) substrate. The most prominent feature of Fig. 6 is the smaller amplitude, about ten times smaller than for air alone (Fig. 4) at $\omega^{+}=1$. This indicates that most of the introduced heat flows directly to the wall. In addition, as the frequency varies, the spacing between the amplitude curves is more uniform in Fig. 6 compared to air alone (Fig. 4). This is another indication that the wall dominates the heat transfer, as frequency defines the thermal penetration perpendicular to the heater. The fluid side is nonuniform with respect to heat penetration because of the velocity distribution. Finally, each curve of Fig. 6 contains a flat portion at low frequency and a falling region at higher frequency. This overall shape is similar to air alone; however, for the air-polymer case, the location of the transition from flat amplitude to falling amplitude occurs at higher Peclet number than for air alone (see Fig. 4). That is, more vigorous convection is needed in order for the heater mounted on a participating wall to be affected by the shear stress.

Figure 7 shows the phase of the spatial-average heater temperature for the air/kapton case plotted versus Peclet number. This is a simulated calibration curve for shear stress by measuring phase. Compared to the phase for the fluid-only case (Fig. 5), the range

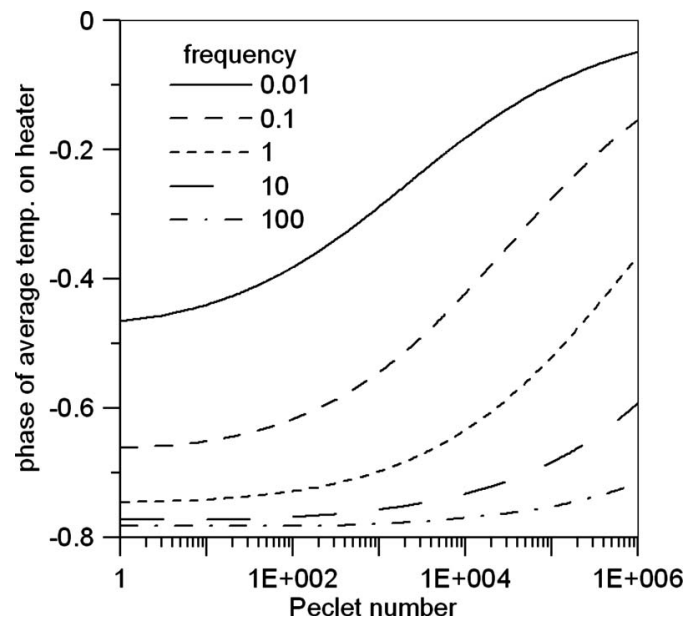

Fig. 7 Phase of spatial-average temperature on the heater for airflow over a polymer wall $\left(k_{1} / k_{2}=0.22, \alpha_{1} / \alpha_{2}=291\right)$ for various frequencies 
Table 1 Typical values of Reynolds and Peclet numbers associated with airflow $(\mathrm{Pr}=0.69)$ in a flat-plate boundary layer, and associated frequency values drawn from Fig. 7

\begin{tabular}{|c|c|c|c|c|c|c|}
\hline $\operatorname{Re}_{x}$ & $x(\mathrm{~m})$ & $a(\mathrm{~m})$ & $\mathrm{Pe}=b a^{2} / \alpha_{f}$ & $u_{\infty}(\mathrm{m} / \mathrm{s})$ & $\omega^{+}$ & $f(\mathrm{~Hz})$ \\
\hline \multicolumn{7}{|c|}{ Laminar $^{\mathrm{a}}$} \\
\hline $10^{3}$ & 0.1 & 0.001 & $7.24\left(10^{-1}\right)$ & $1.59\left(10^{-1}\right)$ & 0.01 & 0.0366 \\
\hline $10^{4}$ & 0.1 & 0.001 & $2.29\left(10^{+1}\right)$ & 1.59 & 0.10 & 0.3660 \\
\hline $10^{5}$ & 0.1 & 0.001 & $7.24\left(10^{2}\right)$ & $1.59\left(10^{1}\right)$ & 1.00 & 3.6600 \\
\hline \multicolumn{7}{|c|}{ Turbulent $^{\mathrm{b}}$} \\
\hline $10^{6}$ & 1.0 & 0.001 & $1.29\left(10^{3}\right)$ & $1.59\left(10^{1}\right)$ & 0.1 & 0.366 \\
\hline $10^{7}$ & 1.0 & 0.001 & $9.31\left(10^{4}\right)$ & $1.59\left(10^{2}\right)$ & 1.0 & 3.660 \\
\hline $10^{8}$ & 1.0 & 0.001 & $6.70\left(10^{6}\right)$ & $1.59\left(10^{3}\right)$ & 10.0 & 36.600 \\
\hline
\end{tabular}

of Pe-sensitive behavior begins at a much higher Peclet number, and the Peclet-sensitive range is much wider. Note the low frequencies involved at a given Peclet number. For frequency $\omega^{+}$ $=1$ the phase varies strongly only for $\mathrm{Pe}>10^{4}$. This normalized frequency corresponds to $\omega=\omega^{+} \alpha_{1} / a^{2}$, which represents $f$ $=\omega /(2 \pi)=3.58 \mathrm{~Hz}$ for a heater with $a=1 \mathrm{~mm}$ in airflow. Making the heater larger should help by increasing the Peclet number (recall $\left.\mathrm{Pe}=b a^{2} / \alpha_{1}\right)$; however, increasing the heater size also increases $\omega^{+}$by the same factor, leaving the phase relationship unchanged. Increasing the heater size should, however, have the effect of increasing the signal size.

A rough estimate of the precision of the phase-based shearstress measurement can be inferred from Fig. 7. At frequency $\omega^{+}=1$, over the shear-stress range $\left(10^{4}<\mathrm{Pe}<10^{5}\right)$, the phase varies over the range $(-0.64,-0.52) \mathrm{rad}$ or $(-36.6,-29.8) \mathrm{deg}$. The average slope over this region is $d(\mathrm{Pe}) / d($ phase $) \approx 13,000 \mathrm{deg}^{-1}$. If the phase can be measured to within $\pm 0.01 \mathrm{deg}$ with a lock-in amplifier, a conservative value [28], then the precision of the shear-stress measurement is approximately 13,000 $\times( \pm 0.01) / 10^{5} \times 100 \%= \pm 0.13 \%$ at $\mathrm{Pe}=10^{5}$. As the calibration curve (Fig. 7) is nonlinear, the precision will vary somewhat with frequency and flow range.

The relationship between dimensionless parameters and a specific airflow geometry is given next. Table 1 contains values for Reynolds and Peclet numbers associated with airflow in a flatplate boundary layer in both laminar and turbulent regimes. The Peclet number depends on the velocity gradient $b$ at the plate surface, which is found from the skin-friction correlations given below the table. The assumed heater size is $1 \mathrm{~mm}$. For each flow situation given in Table 1, a representative frequency is given, drawn from Fig. 7, at which the phase of the observed sensor signal is sensitive to the shear stress. The values in Table 1 show that higher frequencies are associated with higher shear-stress values. The range of frequencies associated with the airflows shown in the table are attainable with conventional electronic equipment.

As indicated above, Fig. 7 is a simulated calibration curve for shear stress found from phase angle. For most sensors such a calibration curve is found from a flow calibration. In the next section a thermal calibration procedure is discussed.

Thermal Calibration. In gas flows, the flow calibration of thermal shear-stress sensors is installation dependent, because the sensor creates a large "heat island" on the wall in which it is mounted [19]. Several researchers have studied in situ flow calibrations $[17,18]$ in an attempt to experimentally compensate for the effects of the wall on the sensor. This section contains a discussion of thermal calibration, which involves analyzing the sensor response without the presence of fluid flow to determine the sensor thermal properties. This is analogous to obtaining the tare weight on a scale or the null reading in a gage. Once the thermal properties of the sensor are obtained, then the sensor flow re- sponse can be interpreted with suitable data-analysis techniques. This idea has previously been explored with a steadily heated sensor [13].

The thermal calibration procedure is essentially the measurement of the thermal properties of the sensor substrate; that the thermal properties of a solid can be reliably measured with a small, periodically heated region is well documented [34,35]. The thermal calibration procedure is the following: Energize the in situ sensor with a steady-periodic signal at Peclet $=0$ (no fluid flow) at several frequencies, measure the sensor response at each frequency, and systematically compare the actual response to the two-body simulated response to determine the wall thermal properties. The wall conductivity $k_{2}$ and the wall diffusivity $\alpha_{2}$ are to be sought.

This procedure for measuring thermal properties is a type of inverse problem. An important part of every multiple-parameter inverse problem are the sensitivity coefficients. For successful estimation of multiple parameters, the sensitivity coefficients must be large and linearly independent (equivalently they must have different shapes when plotted) [36]. For measurement of wall properties, the sensitivity coefficients are derivatives of the data with respect to the sought-after parameters. Let $X$ be the sensitivity coefficient, given by

$$
X_{i}=m_{i} \frac{\partial T}{\partial m_{i}}
$$

where $m_{i}=k_{2}$ or $\alpha_{2}$. The sensitivity coefficients have been computed from the temperature simulation by a finite difference as follows:

$$
X_{i} \approx m_{i} \frac{T\left(m_{i}(1+\epsilon)\right)-T\left(m_{i}\right)}{\epsilon m_{i}}
$$

and are plotted in Figs. 8 and 9 for the air/polymer case. To repeat, the sensitivities must be large and linearly independent for a successful inverse calculation. The amplitudes of the sensitivities, Fig. 8 , become small at higer frequencies, which indicates that there is an upper limit on useful frequencies. Also, at higher frequency, the amplitide of sensitivity tends to have the same descending shape, which is another indication that higher frequencies are not useful. At smaller frequency, the amplitude of the sensitivity has a limiting value, indicating that there is also a lower bound on useful frequencies.

The phases of sensitivities, Fig. 9, are large and of differing shape, especially at the middle-to-higher freqencies shown. Frequencies above $\omega^{+}=100$ probably will not contribute further because both curves flatten out, and any region where the curves have the same shape indicates a lack of linear independence. Frequencies below $\omega^{+}=0.001$ probably do not contribute further be- 


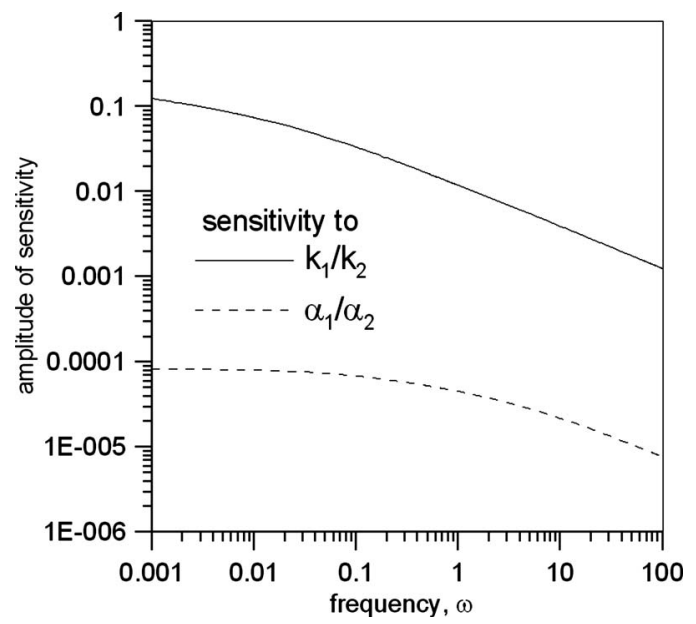

Fig. 8 Amplitude of sensitivity of temperature to thermal properties, computed for the air/polymer case with $\mathrm{Pe}=0$ (no air velocity)

cause one curve approaches zero. These sensitivity curves suggest that for successful measurement of wall properties, data would need to be collected from several frequencies over the range shown.

The sensitivity curves shown here suggest that both conductivity and diffusivity of the wall could be found from both amplitude and phase data. Phase data are probably more important because the phase sensitivity is large, especially at higher frequencies. For further discussion of thermal-property measurements based on phase data, see Refs. [34,37].

There are several ways that thermal calibration could change the way shear-stress sensors are used. For example, some hot-film sensors are factory-built units, which are installed in a cavity on the fluid-flow boundary. If the wall thermal properties are measured pre-install and postinstall, and are unchanged, then the factory calibration could be used for the fluid-flow measurement. As another example, if the in situ wall thermal properties are unchanged before and after a fluid-flow test, then the flow calibration could not have changed during the test. Finally, for situations where flow calibration is inconvenient, such as for high speed, high altitude conditions, the thermal calibration could be combined with the analytical methods discussed in this paper to con-

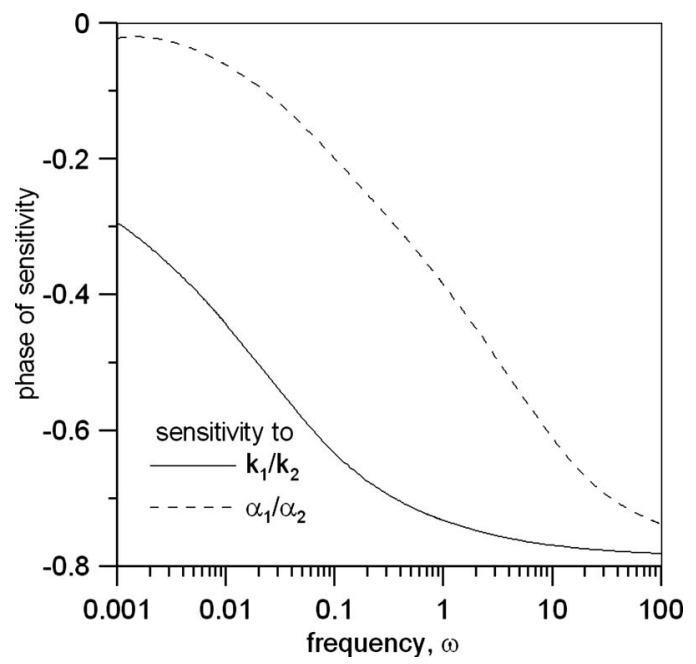

Fig. 9 Phase of sensitivity of temperature to thermal properties, computed for the air/polymer case with $\mathrm{Pe}=0$ (no air velocity) struct simulated calibration curves. The fluid-flow values inferred from this approach would, however, be limited by the veracity of the thermal model of the sensor.

Computing Issues. Considerable programming effort was needed to obtain efficient evaluation of the $\beta$-inversion integral in Eqs. (24) and (25). The CPU time required for the numerical integration was greatly decreased by the recognition that the integrand contains a sinusoidal factor, with zero crossings at $0, \pm 2 \pi$, $\pm 4 \pi$, and so on. The integrand has a maximum magnitude at $\beta$ $=0$ and the magnitude shrinks towards zero as $\beta \rightarrow \pm \infty$. To take advantage of this form, the improper integral on $0<\beta<\infty$ was replaced with a summation of proper integrals, each of width $2 \pi$, beginning at $\beta=0$. Additional terms of this series were added until the fractional change in the magnitude of the running sum was less than a tolerance to provide five-digit precision. The integral over $-\infty<\beta<0$ was handled in a similar way.

Computations were carried out on a Sun Blade 2000 with dual $900 \mathrm{MHz}$ processors running the Solaris operating system. The theory was coded in FORTRAN 77 with variables of type doubleprecision complex. As an example of the computer time required for numerical results, about 20 min was required to compute each curve in Fig. 3, which is composed of 300 points.

Athough the numerical results presented here are based on exact theory, they have been found from numerical integration. Two comments are in order as to whether the results could have been found from a fully numeric calculation (such as finite-difference or finite-element methods). First, fully numeric methods are ill suited to geometries with widely diverse length scales such as this one (thin, small heater on a large domain). A very large number of nodes would be needed near the edges of the heater and many nodes would be needed far from the heater to fill the large domain. Further, since the spatial extent of the thermal boundary layer is both velocity and frequency dependent (see Figs. 2 and 3 ) the nodal mesh would have to be regenerated many times, consuming considerable computer resources, to cover the same range of results given here. Second, the numerical-integration results have been checked against independent limiting cases to four-digit accuracy and were repeatable to five-digit precision; similar accuracy from a fully numeric calculation, if possible at all, would be prohibitively time consuming.

\section{Summary}

The steady-periodic heated film discussed in this paper has several distinct advantages over traditional steadily heated sensors. First, the periodic-heated sensor can be tuned, via frequency, to the shear-stress level of interest. Second, if a lock-in amplifier is used to measure the sensor response, the phase response alone can be used to measure the fluid shear stress, which is important because phase measurements are less sensitive to signal drift caused by electronic amplifiers compared to amplitude measurements. Third, the same lock-in amplifier that gives the flow response can be used for in situ measurement of the sensor thermal properties. This thermal calibration could be used before and after an experiment to verify that the flow calibration has not changed. Finally, for situations where flow calibration is difficult or impossible, the thermal calibration could be used to construct simulated flowcalibration curves based on the analytical techniques presented here.

The present work is limited to steady shear stress. Considerable effort would be needed to include an arbitrary time-varying shear stress, because the analytical advantage of steady-periodic behavior would be lost. One approach is a hybrid scheme in which an analytic solution in the wall could be matched to a fully numeric solution in the fluid flow.

\section{Acknowledgment}

The author gratefully acknowledges support from NASA, Washington DC, under EPSCOR Program Contract No. NCC5- 
165. Thanks also go to Gregg Noffz at NASA Dryden for several valuable discussions of hot-film sensors.

\section{Nomenclature}

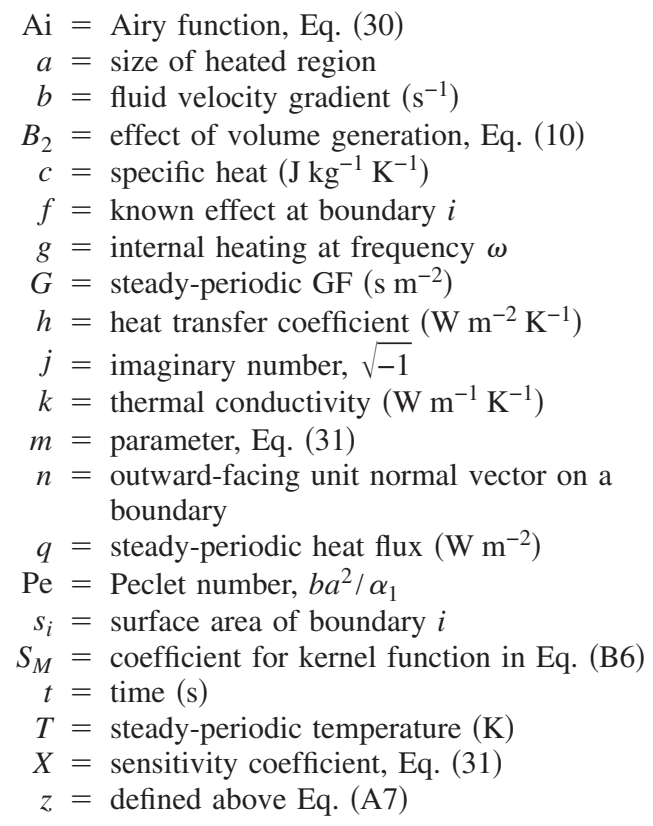

Greek

$$
\begin{aligned}
\alpha & =\text { thermal diffusivity }\left(\mathrm{m}^{2} \mathrm{~s}^{-1}\right) \\
\beta & =\text { wave number, Eq. }(14)\left(\mathrm{m}^{-1}\right) \\
\delta & =\text { Dirac delta function } \\
\nu & =\text { defined for Eq. (B5) } \\
\rho & =\text { defined above Eq. (A6) } \\
\phi & =\text { influence function, Eq. }(20) \\
\omega & =\text { frequency }\left(\mathrm{rad} \mathrm{s}^{-1}\right) \\
\Omega & =\text { domain }
\end{aligned}
$$

\section{Superscripts}

$$
\begin{aligned}
& \sim=\text { time-domain quantity } \\
& -=\text { spatial Fourier transform, Eq. (13) }
\end{aligned}
$$

\section{Subscripts}

$$
\begin{aligned}
1 & =\text { fluid } \\
2 & =\text { solid } \\
h & =\text { heater } \\
i & =\text { index }
\end{aligned}
$$

\section{Appendix A: Fluid-Side Green's Function}

In this appendix the GF for the steady-periodic temperature in a shear flow is developed. The GF associated with the time-periodic problem satisfies the following energy equation (subscript 1 has been supressed in this section):

$$
\frac{j \omega}{\alpha} G+b y \frac{\partial G}{\partial x}=\frac{\partial^{2} G}{\partial x^{2}}+\frac{\partial^{2} G}{\partial y^{2}}+\frac{1}{\alpha} \delta\left(x-x^{\prime}\right) \delta\left(y-y^{\prime}\right)
$$

The boundary conditions are as follows:

$$
\text { at } y=0, \quad \frac{\partial G}{\partial y}=0
$$

$$
\text { at } y \rightarrow \infty, \quad \partial G \text { is bounded }
$$

and

$$
\text { at } x \rightarrow \pm \infty, \quad \partial G \text { is bounded }
$$

A Type 2 boundary (specified flux) is used at $y=0$ to be consistent with the flux condition used in Eq. (10). The solution for $G$ will be found with a Fourier transform along $x$. First, use a simple change of variable to replace $\left(x-x^{\prime}\right)$ with $x$. Then apply Eq. (13) to the above differential equation and boundary conditions to find

$$
\frac{d^{2} \bar{G}}{\partial y^{2}}-\left(\frac{j \omega}{\alpha}+\beta^{2}+\frac{b y j \beta}{\alpha}\right) \bar{G}=-\frac{1}{\alpha} \delta\left(y-y^{\prime}\right)
$$

Let $p=b \beta j / \alpha, \rho=j \omega / \alpha+\beta^{2}+p y$. Then the differential equation can be written as

$$
\frac{d^{2} \bar{G}}{\partial y^{2}}-\rho \bar{G}=-\frac{1}{\alpha} \delta\left(y-y^{\prime}\right)
$$

This second-order, ordinary differential equation can be transformed into the Airy equation with a change of variable to $z$ $=\rho / p^{2 / 3}$. The solution to this equation will be sought by splitting the domain at $z=z^{\prime}$ into two regions. That is, we seek solutions

$$
\begin{gathered}
\frac{d^{2} \bar{G}_{a}}{\partial z^{2}}-z \bar{G}_{a}=0, \quad z_{0}<z<z^{\prime} \\
\frac{d^{2} \bar{G}_{b}}{\partial z^{2}}-z \bar{G}_{b}=0, \quad z^{\prime}<z<\infty \\
\text { where } z_{0}=\left.z\right|_{y=0} \\
\text { and } z^{\prime}=\left.z\right|_{y=y^{\prime}}
\end{gathered}
$$

The general solution is given by

$$
\begin{aligned}
& \bar{G}_{a}(z)=C_{1} \operatorname{Ai}(z)+C_{2} \operatorname{Bi}(z) \\
& \bar{G}_{b}(z)=C_{3} \operatorname{Ai}(z)+C_{4} \operatorname{Bi}(z)
\end{aligned}
$$

where $\operatorname{Ai}(z)$ and $\operatorname{Bi}(z)$ are Airy functions (Ref. [38], p. 448) and $C_{j}$ are constants. As there are four undetermined constants, four conditions are needed. Two are boundary conditions on $y$, restated here in terms of $z$,

$$
\begin{gathered}
\text { at } z=z_{0}, \quad \frac{\partial \bar{G}_{a}}{\partial z}=0 \\
\text { at } z \rightarrow \infty, \quad \bar{G}_{b} \text { is bounded }
\end{gathered}
$$

A matching condition is required at $z=z^{\prime}$, that is,

$$
\bar{G}_{a}\left(z^{\prime}\right)=\bar{G}_{b}\left(z^{\prime}\right)
$$

There is also a jump condition at $z=z^{\prime}$, found by integrating Eq. (A6) over $\left(y^{\prime}-\epsilon, y^{\prime}+\epsilon\right)$, taking the limit as $\epsilon \rightarrow 0$, and converting $d / d y$ to $d / d z$ with the chain rule as follows:

$$
\left.\frac{d \bar{G}_{b}}{\partial z}\right|_{z=z^{\prime}}-\left.\frac{d \bar{G}_{a}}{\partial z}\right|_{z=z^{\prime}}=-\frac{1}{\alpha p^{1 / 3}}
$$

With these four conditions the four constants may be determined algebraically and substituted into the general solution. The GF for a Type 2 boundary at $y=0$ is given by

$$
\bar{G}\left(z, z^{\prime}\right)=\left\{\begin{array}{l}
{\left[\operatorname{Ai}^{\prime}\left(z_{0}\right) \operatorname{Bi}(z)-\operatorname{Bi}^{\prime}\left(z_{0}\right) \operatorname{Ai}(z)\right] \frac{\operatorname{Ai}\left(z^{\prime}\right)}{\alpha p^{1 / 3} D}, \quad z<z^{\prime}} \\
{\left[\operatorname{Ai}^{\prime}\left(z_{0}\right) \operatorname{Bi}\left(z^{\prime}\right)-\operatorname{Bi}^{\prime}\left(z_{0}\right) \operatorname{Ai}\left(z^{\prime}\right)\right] \frac{\operatorname{Ai}(z)}{\alpha p^{1 / 3} D}, \quad z>z^{\prime}}
\end{array}\right.
$$

where

$$
D=\left[\operatorname{Bi}^{\prime}\left(z^{\prime}\right) \mathrm{Ai}\left(z^{\prime}\right)-\operatorname{Ai}^{\prime}\left(z^{\prime}\right) \operatorname{Bi}\left(z^{\prime}\right)\right] \operatorname{Ai}^{\prime}\left(z_{0}\right)
$$

As expected, this GF satisfies reciprocity: $\bar{G}\left(z, z^{\prime}\right)=\bar{G}\left(z^{\prime}, z\right)$.

Special case for heating at the wall. The special case of the fluid response to heating at the wall has been published previously [7]. In this case, $z^{\prime} \rightarrow z_{o}$, and the expression for $\bar{G}$ simplifies to 


$$
\bar{G}\left(z, z_{0}\right)=-\frac{1}{\alpha p^{1 / 3}} \frac{\mathrm{Ai}(z)}{\mathrm{Ai}^{\prime}\left(z_{0}\right)}
$$

This is the fluid-side GF used in the numerical examples.

The complex-valued Airy function needed for this GF was computed from series expressions. Expressions for $\operatorname{Ai}(z)$ and $\mathrm{Ai}^{\prime}(z)$ for small- $z$ and large- $z$ values (Ref. [38], pp. 446-448) were coded in FORTRAN and spot-checked against a commercial mathematics code. Numerical values were highly accurate at small- $|z|$ and large- $|z|$, with a minimum of five-digit accuracy at intermediate values near $|z|=5$.

\section{Appendix B: Solid-Side Green's Function}

The GF for the steady-periodic temperature in the solid satisfies the following differential equation (subscript 2 has been suppressed in this section):

$$
\frac{j \omega}{\alpha} G=\frac{\partial^{2} G}{\partial x^{2}}+\frac{\partial^{2} G}{\partial y^{2}}+\frac{1}{\alpha} \delta\left(x-x^{\prime}\right) \delta\left(y-y^{\prime}\right)
$$

The boundary conditions are as follows:

$$
\begin{gathered}
\text { at } x \rightarrow \pm \infty, \quad \partial G \text { is bounded } \\
\text { at } y=0, \quad \frac{\partial G}{\partial y}=0 \\
\text { at } y=W, \quad k \frac{\partial G}{\partial y}+h G=0
\end{gathered}
$$

For the semi-infinite body, replace the condition at $y=W$ with a boundedness condition at $(y \rightarrow \infty)$. For the present work, the spatial-Fourier transform of this GF is needed. First use a simple change of variable to replace $\left(x-x^{\prime}\right)$ with $x$. Then apply the Fourier transform, Eq. (13), to the above differential equation, to find

$$
\frac{d^{2} \bar{G}}{\partial y^{2}}-\nu^{2} \bar{G}=-\frac{1}{\alpha} \delta\left(y-y^{\prime}\right)
$$

where $\nu^{2}=\beta^{2}+j \omega / \alpha$. The solution for this GF in Fourier space is given by [32]

$$
\begin{aligned}
\bar{G}\left(y, y^{\prime}, \nu\right)= & \frac{S_{2}^{-}\left(e^{-\nu\left(2 W-\left|y-y^{\prime}\right|\right)}+e^{-\nu\left(2 W-y-y^{\prime}\right)}\right)}{2 \alpha \nu\left(S_{2}^{+}-S_{2}^{-} e^{-2 \nu W}\right)} \\
& +\frac{S_{2}^{+}\left(e^{-\nu\left(\left|y-y^{\prime}\right|\right)}+e^{-\nu\left(y+y^{\prime}\right)}\right)}{2 \alpha \nu\left(S_{2}^{+}-S_{2}^{-} e^{-2 \nu W}\right)}
\end{aligned}
$$

Coefficients $S_{2}^{+}$and $S_{2}^{-}$depend on the type of boundary condition at Side $2(y=W)$ and are given by

$$
\begin{gathered}
S_{2}^{+}=\left\{\begin{array}{cc}
1 & \text { Type } 0, \text { Type } 1, \text { or Type } 2 \\
k \nu+h & \text { Type } 3
\end{array}\right. \\
S_{2}^{-}=\left\{\begin{array}{cc}
0 & \text { Type } 0 \\
-1 & \text { Type } 1 \\
1 & \text { Type } 2 \\
k \nu-h & \text { Type } 3
\end{array}\right.
\end{gathered}
$$

To find the GF in $x$-space an inverse-Fourier transform is needed. However, in the present work the GF is used to find the temperature in Fourier transform space and then the inverse-Fourier transform is applied to the temperature solution.

Special case for heating at $y=0$. In the special case with localized heating at the fluid-solid surface, the GF may be simplified. Replace $y^{\prime}=0$ with the above expression to find

$$
\bar{G}(y, 0, \nu)=\frac{S_{2}^{-}\left(e^{-\nu(2 W-|y|)}+e^{-\nu(2 W-y)}\right)}{2 \alpha \nu\left(S_{2}^{+}-S_{2}^{-} e^{-2 \nu W}\right)}+\frac{S_{2}^{+}\left(e^{-\nu(|y|)}+e^{-\nu y}\right)}{2 \alpha \nu\left(S_{2}^{+}-S_{2}^{-} e^{-2 \nu W}\right)}
$$

Finally, for the solution to the conjugate problem, the temperature is also evaluated at the fluid-solid interface. Replace $y=0$ with the above expression to obtain

$$
\bar{G}(0,0, \nu)=\frac{S_{2}^{+}+S_{2}^{-} e^{-2 \nu W}}{\alpha \nu\left(S_{2}^{+}-S_{2}^{-} e^{-2 \nu W}\right)}
$$

This expression, with the appropriate coefficients given above, is the solid-side GF used in the numerical examples.

\section{References}

[1] Ludwieg, H., 1950, "Instrument for Measuring the Wall Shearing Stress of Turbulent Boundary Layers," NACA Report No. TN 1284.

[2] Mayer, R., Henkes, R. A. W. M, and Van Ingen, J. L., 1988, "Quantitative Infrared Thermography for Wall Shear Stress Measurement in Laminar Flow," Int. J. Heat Mass Transfer, 41, pp. 2347-2360.

[3] Baughn, J. W., Mayhew, J. E., Butler, R. J., Byerley, A. R., and Rivir, R. B., 1999, "Turbine Blade Flow Separation and Reattachment at Low Reynolds Number," ASME J. Heat Transfer, 121(3), poster insert.

[4] Stone, H. A., 1989, "Heat/Mass Transfer From Surface Films to Shear Flows at Arbitrary Peclet Numbers," Phys. Fluids A, 1(7), pp. 1112-1122.

[5] Phillips, C. G., 1990, "Heat and Mass Transfer From a Film Into Steady Shear Flow," Q. J. Mech. Appl. Math., 43, pp. 135-160.

[6] Soliman, M., and Chambre, P. L., 1967, "On the Time-Dependent Leveque Problem," Int. J. Heat Mass Transfer, 10, pp. 169-179.

[7] Geshev, P. I., 1996, "The Green Function Method for Calculating Characteristics of a Small Strip-Shaped Shear Stress Probe," J. Electroanal. Chem., 410, pp. $1-8$.

[8] Steenhoven, A. A., and van de Beuken, F. J. H. M., 1991, "Dynamical Analysis of Electrochemical Wall Shear Rate Measurements," J. Fluid Mech., 231, pp. 599-614.

[9] Cole, K. D., 1997, "Conjugate Heat Transfer From a Small Heated Strip," Int. J. Heat Mass Transfer, 40(11), pp. 2709-2719.

[10] Harman, S., 1998, "Conjugate Heat Transfer: Effects of Axial Conduction and the Layered Wall," MS thesis, University of Nebraska, Mechanical Engineering Department, Lincoln, NE.

[11] Stein, C. F., Johansson, P., Bergh, J., Löfdahl, L., Sen, M., and Gad-el-Hak, M., 2002, "An Analytical Solution to a Conjugate Heat Transfer Problem," Int J. Heat Mass Transfer, 45(12), pp. 2485-2500.

[12] Chao, J. H., Shyy, W., Thakur, S. S., Sheplak, M., and Mei, R. W., 2005, "Effect of Conjugate Heat Transfer on MEMS-Based Thermal Shear Stress Sensor," Numer. Heat Transfer, Part A, 48(3), pp. 197-217.

[13] Cole, K. D., and Beck, J. V., 1988, "Conjugated Heat Transfer From a Hot Film Probe for Transient Air Flow," ASME J. Heat Transfer, 110, pp. 290 296.

[14] Liang, P. W., and Cole, K. D., 1992, "Conjugated Heat Transfer From a Rectangular Hot-Film With the Unsteady Surface Element Method," J. Thermophys. Heat Transfer, 6, pp. 349-355.

[15] Cole, K. D., 2002, "Analysis of Pulse Heating on a Fluid-Cooled Surface for Fluid Shear Stress," Proceedings, International Mechanical Engineering Congress and Exposition, New Orleans, LA, Paper No. 2-20-5-5.

[16] Dinu, C., Beasley, D. E., and Figliola, R. S., 1998, "Frequency Response Characteristics of an Active Heat Flux Gage," ASME J. Heat Transfer, 120, pp. 557-582.

[17] Bellhouse, D. J., and Schultz, D. S., 1967, "The Determination of Flucuating Velocity in Air With Heated Thin-Film Gages," Phys. Fluids, 29, pp. 289-295.

[18] Alfredsson, P. H., Johansson, A. V., Haritonidis, J. H., and Eckelmann, H. 1988, "On the Fluctuating Wall Shear Stress and the Velocity Field in the Viscous Sublayer," Phys. Fluids, 31, pp. 1026-1033.

[19] Reda, D. C., 1991, "Rise-Time Response of Nickel-Foil-on-Kapton-Substrate, Hot-Film Shear-Stress Sensors," Proceedings of the 29th Aerospace Sciences Meeting, Reno, NV, Paper No. AIAA-91-0169.

[20] Tardu, F. S., and Pham, C. T., 2005, "Response of Wall Hot-Film Gages With Longitudinal Diffussion and Heat Conduction to the Substrate," ASME J. Heat Transfer, 127, pp. 812-819.

[21] Gustafsson, S. E., Chohan, M. A., Ahmed, K., and Maqsood, A., 1984, "Thermal Properties of Thin Insulating Layers Using Pulse Transient Hot Strip Measurements," J. Appl. Phys., 55(9), pp. 3348-3353.

[22] Zhong, Q. Y., Favro, L. D., and Thomas, R. L., 2000, "Thermal Wave Reflections of a Pulsed Stripe Heat Source From a Plane Boundary," J. Appl. Phys., 87, pp. 3999-4004.

[23] Cahill, D. G., 1990, "Thermal Conductivity Measurement From 30 to $750 \mathrm{~K}$ : the $3 \omega$ Method," Rev. Sci. Instrum., 61, pp. 802-808.

[24] Cole, K. D., and McGahan, W. A., 1993, Theory of Multilayers Heated by Laser Absorption, ASME J. Heat Transfer, 115, pp. 767-771.

[25] Hu, H., Wang, X., and Xu, X., 1999, "Generalized Theory of the Photoacoustic Effect in a Layered Material,” J. Appl. Phys., 86, pp. 3953-3958.

[26] Bonne, U., and Kubisiak, D., 2001, "Actuation-Based Microsensors,” Smart 
Mater. Struct., 10(6), pp. 1185-1195.

[27] Al-Salaymeh, A., and Durst, F., 2004, "Development and Testing of a Novel Single-Wire Sensor for Wide Range Flow Velocity Measurements," Meas. Sci. Technol., 15(5), pp. 777-788.

[28] Chung, W. S., Kwon, O., Choi, D. S., Park, S., Choi, Y. K., and Lee, J. S., 2004, "Tunable AC Thermal Anemometry," Superlattices Microstruct., 35(36), pp. 325-338.

[29] Rachalski, A., 2006, "High-Precision Anemometer With Thermal Wave," Rev. Sci. Instrum., 77(9), p. 095107.

[30] Mandelis, A., 2001, Diffusion-Wave Fields, Mathematical Methods and Green's Functions, Springer, New York.

[31] Beck, J. V., Cole, K. D., Haji-Sheikh, A., and Litkouhi, B., 1992, Heat Conduction Using Green's Functions, Hemisphere, New York.

[32] Cole, K. D., 2006, "Steady-Periodic Green's Functions and ThermalMeasurement Applications in Rectangular Coordinates," ASME J. Heat Trans- fer, 128(7), pp. 709-716.

[33] White, F. M., 1999, Fluid Mechanics, 4th ed., McGraw-Hill, New York.

[34] Haji-Shiekh, A., Hong, Y. S., You, S. M., and Beck, J. V., 1998, "Sensitivity Analysis for Thermophysical Property Measurements Using the Periodic Method," ASME J. Heat Transfer, 120(3), pp. 568-576.

[35] Aviles-Ramos, C., Haji-Shiekh, A., Beck, J. V., and Dowding, K. J., 2001 , "Estimation of Thermophysical Properties by the Spectral MethodDevelopment and Evaluation," ASME J. Heat Transfer, 123(1), pp. 24-30.

[36] Dowding, K. J., and Blackwell, B. F., 2001, "Sensitivity Analysis for Nonlinear Heat Conduction," ASME J. Heat Transfer, 123, pp. 1-10.

[37] Cole, K. D., 2004, "Analysis of Photothermal Characterization of Layered Materials-Design of Optimal Experiments," Int. J. Thermophys., 25, pp. 1567-1584.

[38] Abramowitz, M., and Stegun, I., 1964, Handbook of Mathematical Functions, Dover, New York 